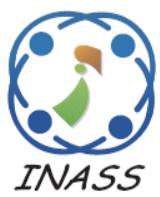

\title{
Image Analysis for MRI Based Brain Tumour Detection Using Hybrid Segmentation and Deep Learning Classification Technique
}

\author{
Sudheesh Kannur Vasudeva Rao ${ }^{1 *}$ \\ Basavaraj Lingappa ${ }^{1}$ \\ ${ }^{I}$ Department of Electronics and Communication Engineering, \\ Academy for Technical and Management Excellence College of Engineering, Mysuru, Karnataka, India \\ * Corresponding author's Email: sudheesh.vvce@gmail.com
}

\begin{abstract}
Indefinite and uncontrollable growth of cells leads to tumours in the human brain. A proper treatment and early diagnosis of brain tumours are essential to prevent permanent damage to the brain. In medical image diagnosis, the tumour segmentation and classification schemes are used for identifying the tumour and non-tumour cells in the brain. The automatic classification is a challenging task which utilizes the traditional methods due to its more execution time and ineffective decision making. To overcome this problem, this research proposes an automatic tumour classification method named as Hybrid Kernel based Fuzzy C-Means clustering - Convolutional Neural Network (Hybrid KFCM-CNN) method. The performance of Hybrid KFCM- CNN method is validated using T1-Weighted Contrast Enhanced Magnitude Resonance Imaging (T1 -W CEMRI) database. A tumour portion is segmented from the MRI brain image by using Hybrid KFCM method. After the segmentation process, statistical features and super pixels based SIFT, Discrete Cosine Transform (DCT) methods were performed on the segmented image to enhance feature subsets. The best feature values were given to the $\mathrm{CNN}$ classifier as an input; it is classified into different types of brain tumours such as Meningioma, Glioma and Pituitary. The experimental outcome shows that the Hybrid KFCMCNN method improved the accuracy of brain tumour classification up to $14.06 \%$ than existing classifiers: SVM and CNN.
\end{abstract}

Keywords: Brain tumour, Classification, Convolution neural network, Kernel based fuzzy c-means, Segmentation.

\section{Introduction}

Brain tumour detection is significant algorithm for identifying the tumour in brain [1]. Brain tumour is an abnormal cell growth in the human brain; it is classified into two types such as benign and malignant. The benign type of tumour does not spread to other organs in the human body, so it can be treated by surgical removal. The malignant type of tumour it generally spreads to other organs and tissues [2]. Brian tumour treatment process depends on the physician's knowledge and experience. For this reason, utilizing an Automated Tumour Detection System (ATDS) is important to help physicians and radiologists to identify brain tumours $[3,4]$. The early diagnosis and appropriate treatment of brain tumour are essential to prevent permanent damage the brain [5]. Computer Aided Detection
(CAD) of the brain tumour is an efficient tool for noninvasively brain tumour $[6,7]$. The iron region is extracted in the brain by an FCM approach. These regions are visible in MRI medical image compared to the output of the K-means images. Although the fuzzy c-means requires more computation time [8]. An unsupervised segmentation technique automatically partitions the images without operator intervention. However, these types of techniques implemented using prior knowledge at the design time [9]. Clustering is one of the unsupervised learning algorithms to segment colour images, but it's not possible to perform well for various types of images [10]. The detection of the tumour regions is a challenging task in tumour MRI image because of its low sensitive boundary pixels [11]. Non-awareness of patterns of KFCM is solved by extreme learning machine algorithm and artificial bee colony algorithm used for minimizing the membership 
subspace plane structure [12]. KFCM segmentation method is better for segmentation of tumour segmentation region [13]. However, the detection and diagnosis of tumour detection still needs more in classifying the tumours.

Therefore this research proposes the automatic classification method for classify the brain tumour based on MRI medical image. Initially, the pixels present in the individual modality of the MRI image is applied to Hybrid KFCM to achieve best clustering. The proposed method uses a Hybrid KFCM algorithm to segment the clusters after that the Otsu's threshold value is applied to the segmented image for best clustering selection. After segmentation, the high (SIFT and wavelet feature) and low-level features (mean, standard deviation and variance) are extracted based on super pixel SIFT algorithm. The robust information of input, segmented and feature extraction images are given to CNN classifier for obtaining the tumour level of MRI images.

This research paper is composed as follows; Section 2 presents an extensive survey of recent papers on brain tumour detection techniques. Section 3 briefly described an effective Hybrid KFCM-CNN methodology. Section 4 shows the comparative experimental result of the existing and Hybrid KFCM-CNN method. The conclusion is made in Section 5.

\section{Literature survey}

The researchers have suggested a number of segmentation and classification techniques for brain tumour detection in the medical field. A brief evaluation of a few significant segmentation and classification techniques are presented in this section.

Md. Sujan, N. Alam, S. A. Noman, and M. Jahirul Islam [14] proposed a new technique based on thresholding with the morphological image analysis to identify a brain tumour. Initially, the colour image converted to a grayscale image, after that noise were eliminated from the grayscale image by using a filtering approach. The gray scale image was converted to the binary image, adding 0.3 threshold values with Otsu's threshold value for accurately segment the tumour portion. Next, the morphological operation performed to detect the brain tumour that consists of the brightest region of the Magnitude Resonance Image (MRI). The proposed method was achieved $84.72 \%$ of accuracy for only 61 patient's brain tumour detection segmentation process. So, it may be not suitable for large datasets.

D. Ravi, H. Fabelo, G. M. Callico, and G. Yang [15] proposed a new dimensionality reduction scheme used for tumour classification map. The proposed method consists of two steps such as dimensionality reduction using t-distributed stochastic neighbour and classification using Semantic Texton Forest (STF). The main advantage of the proposed method was minimized risk of the disease recurrence. Experimental results show that the proposed method well performed than the existing method in terms of specificity and sensitivity. However, the proposed dimensionality minimization method was highly affected by the number of samples in the training set. When the number of samples was low, the detection rate will be reduced.

A. Raju, P. Ratna, Suresh, and R. Rajeswara Rao [16] proposed Bayesian Fuzzy Clustering (BFC) technique based brain tumour and the brain tumour classification was done by HCS optimization algorithms based on SVNN classifier. This classification method determines the level of the brain tumour by using the features of the segments generated by BFC algorithm. The proposed automatic method of the classification achieved the accuracy of 0.93 , the specificity of 0.99 , sensitivity of 0.93, respectively. Hence, this proposed classification algorithm used for efficient decision making by physicians. This work requires preprocessing approach to further improve the land cover classification accuracy.

K. Usman, and K. Rajpoot [17] proposed a brain tumour segmentation, and classification scheme for Multi-modality MRI scans. From the pre-processed images, intensity, local neighbourhood, intensity variance and the wavelet texture were extracted. Subsequently the integrated features were provided to the random forest classifier to predict five classes such as back ground, necrosis, edema non-enhancing tumour and enhancing tumour. These class labels were used to hierarchically compute 3 different regions in terms of complete tumour, active tumour and enhancing tumour. This proposed method achieved $88 \%$ dice overlap for the complete tumour region, $75 \%$ of the core tumour region and $95 \%$ for enhancing tumour region. However, this method provided poor robustness in large datasets.

M. Soltaninejad, G. Yang, T. Lambrou, N. Allinson, T. L. Jones, T. R. Barrick, F. A. Howe, and $X$. Ye [18] implemented an automatic scheme for detection and segmenting the brain tumours by using Super Pixel based Extremely Randomness Trees (SPERTs) technique. This technique was very fast, more reproducible and provided the delineation that supported the patient management. The intensity, Gabor Texton, fractal features were analysed and the curvature was computed from each super-pixel within the entire brain region in flair MRI to confirm 


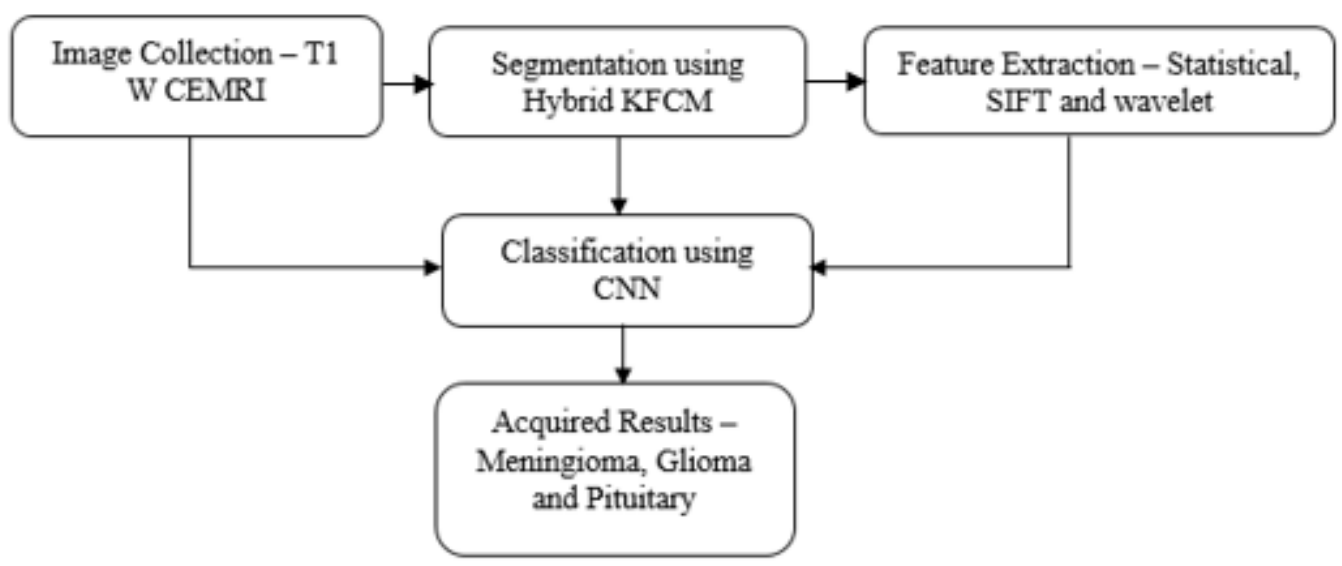

Figure. 1 Block diagram of the Hybrid KFCM-CNN method

robust classification. Extremely Randomized Trees (ERT) classifier was compared with Support Vector Machine (SVM) to classify each super pixel into tumour and non-tumour. However, the Texton feature is not straightforward to provide an accurate number of structures presented in image.

In this research, the Hybrid KFCM-CNN method is implemented to overcome the above-mentioned limitations and to improve the performance of the automatic brain tumour detection.

\section{Hybrid KFCM-CNN methodology}

In this scenario, the MRI image is segmented using hybrid KFCM algorithm for detecting mass tumour in the brain. This hybrid KFCM retains the more information of the original image to detect tumour cells accurately compared to other segmentation techniques. The identification of the edema growth is performed employing the MRI images. The MRI images are processed and that allows the proposed automatic Hybrid KFCM-CNN method for tumour detection.

The main objective of the proposed automatic method is to maximize classification accuracy in brain tumour detection by using Hybrid KFCM-CNN method. The proposed Hybrid KFCM-CNN method has three steps segmentation, feature extraction, and classification. The block diagram of the Hybrid KFCM-CNN method is shown in Fig. 1. The brain image of the individual patient is stored as four various types of modalities such as $\mathrm{T} 1, \mathrm{~T} 2, \mathrm{~T} 1 \mathrm{C}$ and flair. Every modality comprises of a huge number of slice; these modalities are segmented utilizing hybrid KFCM algorithm. Next, the segments are subject to feature extraction by employing the statistical, wavelet transform and SIFT. The extracted features are much more robust, effective in locating the cancer cells and classifies tumour types. The classifier yields

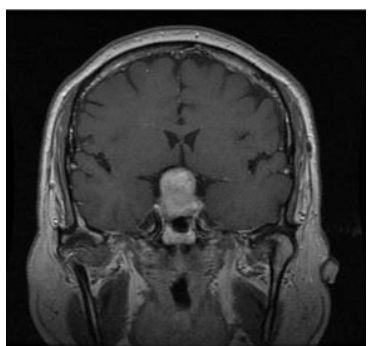

(a)

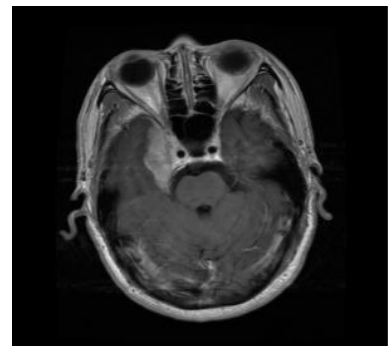

(b)

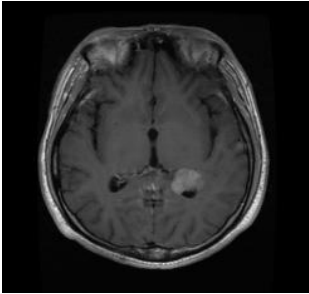

(c)

Figure. 2 Input image: (a) Meningioma input image, (b) Glioma input image, and (c) Glioma input image

the tumour type information as meningioma, Glioma and Pituitary. This research considers an MRI image with various modalities in terms of T1, T2, T3 and T4. Every modalities comprises of slices, which is represented in Eq. (1).

$$
s=\left\{s_{1}^{i}, s_{2}^{i}, \ldots ., s_{n}^{i}\right\} ; 1 \leq i \leq 4
$$

Here, $i$ is a total modality present in the MRI brain image and $s$ represents number of slices. Fig. 2 shows the input image of different brain tumour regions.

\subsection{Segmentation using Hybrid KFCM clustering technique.}

In past years, an integrated the K-means clustering with FCM system and this integrated hybrid clustering method used for automatic brain tumour segmentation. This method significantly reduced the computation time of image segmentation by the combination of K-means clustering and FCM. 
The experiment is carried-out on publicly available datasets: Brain (DS1), (DS2), and (DS3) in order to validate the accuracy of the proposed method [19]. Developed a new system for multi-resolution MRI image segmentation by a morphological pyramid with FCM clustering. Initially, Wavelet MultiResolution (WMR) used to manage the spatial context between the pixels. Next, Morphological pyramid is used to fuse resulting multi-resolution images with original image to maximize sharpness and to reduce noise in the processed image. At the final stage, the FCM was used for image segmentation [20]. The fundamental reason behind inappropriate segmentation happens during the processing of a noisy image by FCM. The KFCM is used to solve the problem of FCM. In this research, the hybrid KFCM is used for segmenting the tumour regions using MRI images. This hybrid KFCM algorithm uses the heterogeneity of the grayscales in the neighbourhood for computing the local contextual information that obtain a new class of distance measure based on hyper tangent function is represented Eq. (2).

$$
M\left(a_{k}, b_{i}\right)=1-\tan m\left(\frac{-\left\|a_{k}-b_{i}\right\|^{2}}{\sigma^{2}}\right)
$$

Here, $a_{k}$ is a finite set of $N$ data points belonging to the cluster $b_{i}$.C is cluster, and $\sigma$ represents the user supplied parameter. The significant objective function is given in Eq. (3).

$$
R_{h}=\sum_{k=1}^{N} \sum_{i=1}^{c} q_{k i}^{h}\left\|\varphi\left(a_{k}\right)-\varphi\left(b_{i}\right)\right\|^{2}
$$

Here, $q_{k i}$ is the fuzzy membership of the $k^{\text {th }}$ data point with respect to cluster $i$ and $h$ denotes weighting exponent $1 \leq h<\infty . \varphi$ is mapping function. Here, use the Gaussian kernel function for non-linear mapping of image plane into linear high Dimensional Future Space (DFS). In hybrid KFCM $\left\|\varphi\left(a_{k}\right)-\varphi\left(b_{i}\right)\right\|^{2}=2\left(1-M\left(a_{k}, b_{i}\right)\right.$ and a new version objective function using above equation is represented in Eq. (4).

$$
R_{h}=2 \sum_{k=1}^{N} \sum_{i=1}^{c} q_{k i}^{h}\left(1-M\left(a_{k}, b_{i}\right)\right)
$$

Here, membership function $\left(q_{k i}\right)$ and uploading centroid $\left(b_{i}\right)$ is done by utilizing Eq. (5) and (6).

$$
q_{k i}=\frac{\left(\frac{1}{1-M\left(a_{k}, b_{i}\right)}\right)^{(1 / h-1)}}{\sum_{j=1}^{c}\left(1-M\left(a_{k}, b_{j}\right)\right)^{(1 / h-1)}}
$$
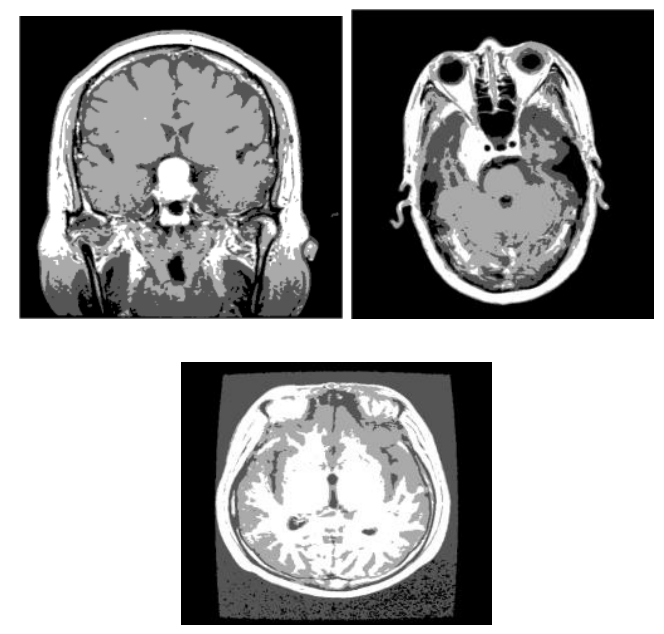

Figure. 3 Clustered of three brain tumour images (Meningioma, Glioma and Pituitary)
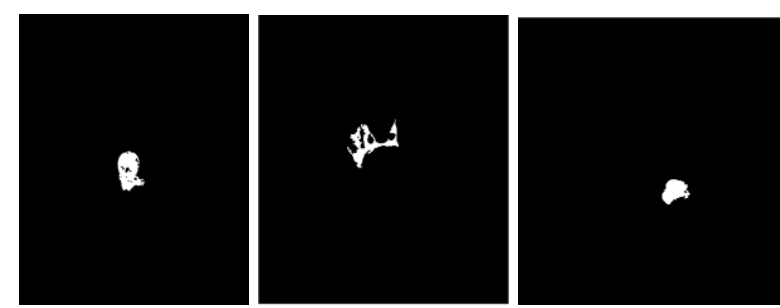

Figure. 4 Segmented of three brain tumour images

$$
b_{i}=\frac{\sum_{k=1}^{N}\left(q_{k i}^{h} M\left(a_{k}, b_{i}\right)\left(1+\tan m\left(-\left\|\frac{a_{k}-b_{i}}{\sigma^{2}}\right\|^{2}\right)\right) a_{k}\right)}{\sum_{k=1}^{N} q_{k i}^{h} M\left(a_{k}, b_{i}\right)\left(1+\tan m\left(-\left\|\frac{a_{k}-b_{i}}{\sigma^{2}}\right\|^{2}\right)\right)}
$$

Finally, the Otsu's threshold algorithm applies to segmented clusters for best clustering selection. Fig. 3 shows clustered image of three brain tumour regions. Fig. 4 shows the segmented three brain tumour images.

\subsection{Feature extraction}

The typical features of an image are used for detection of tumour. The features are extracted from the segmented regions of the image by using statistics, wavelet transform and superpixels based SIFT algorithm.

\subsubsection{Statistical feature}

The gray level values of an image give spatial features and that can be utilized in bio-logical applications to learn the features of the image. In this research, statistical feature detecting and analysis can improve the diagnosis, various stage of tumour ad therapy response assessment. The statistical feature formula used for some of the useful features is listed as follows.

Mean: Mean of the image depends on the homogeneity of a brightness of the MRI image. If the 
image is determinedly bright while mean value is high. The mean is defined as the sum of all pixel values separated by sum of pixels. It gives the average distribution of the intensity value of the image. The Eq. (7) is expressed as the mean value.

$$
\text { Mean }=\frac{1}{p \times q} \sum_{a=0}^{p-1} \sum_{b=0}^{q-1} f(a, b)
$$

Here, $p \times q$ and $f(a, b)$ is original and decomposed image.

Variance: The variance characteristic distribution of the computed gray levels. If there is a variance between gray level values of mean after that variance will be maximized. The variance used for computing each pixel differs from the mean value that is given as the average of the square of the difference between mean and individual pixels. The variance is computed by using Eq. (8).

$$
\text { Variance }=\frac{1}{p \times q} \sum_{a=0}^{p-1} \sum_{b=0}^{q-1}(f(a, b)-M e a n)^{2}
$$

Standard Deviation: This defines the difference in set of data value form the mean, it can be described as the square root of variance. The standard deviation is given Eq. (9).

$$
\begin{gathered}
\text { Standard Deviation }= \\
\sqrt{\frac{1}{p \times q} \sum_{a=0}^{p-1} \sum_{b=0}^{q-1}(f(a, b)-\text { Mean })^{2}}
\end{gathered}
$$

\subsubsection{Discrete cosine transform}

DCT is a transform to extract appropriate features for tumor detection. The DCT has the efficient property that used for a typical image to visually significant the information which is focused in some coefficients. The coefficient can be employed as a type of signature that is useful for brain tumour segmentation tasks. The DCT of $X \times Y$ gray scale matrix of the tumor image $f(a, b)$ is expressed in Eq. (10).

$$
\begin{gathered}
P(a, b)=\sum_{a=0}^{X-1} \sum_{b=0}^{Y-1} f(a, b) \propto(a) \propto(b) \times \\
\cos \left[\frac{(2 a+1) a \pi}{2 X}\right] \operatorname{Cos}\left[\frac{(2 b+1) b \pi}{2 Y}\right]
\end{gathered}
$$

Here, equation calculates the one entry of $(a, b)^{t h}$ transformed image from the pixel value of original image matrix. The values $P(a, b)$ are the DCT coefficients. This DCT is effective for the small
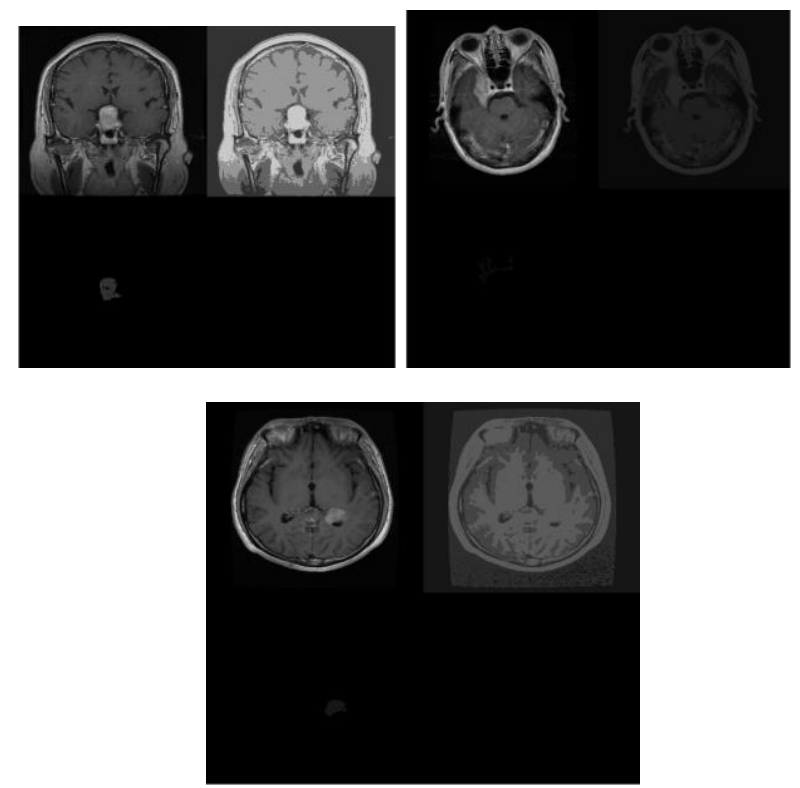

Figure. $5 \mathrm{CNN}$ training images of three different tumours

square inputs as image blocks of $8 \times 8$ pixels. After applying DCT to entire tumour MR image, low frequency band feature is extracted.

\subsubsection{Super pixel based SIFT feature}

The geometrical features are extracted by using a super pixel based SIFT feature extraction algorithm. The SIFT provides the image of object that are unaffected by object scaling and rotation. This SIFT algorithm consists of four filtering schemes such as Scale Space Extrema Detection (SSED), Key Point Localization (KPL), Orientation Assignment (OA) and Key Point Descriptor (KPD). In SSED, the filter detects the space location and scale from various views of the objects. This SSED is used to obtain the scale space function based on Gaussian function. In KPL, to eliminate more key points and to detect the edges the Laplacian is computed. The OA scheme targets to assign a consistent orientation to the key points established based on local image properties. The local gradient statistics help to create key point's descriptors. The gradient information is rotated for grouping with the orientation of the key points. The output vectors are SIFT key points, which are dependent on the nearest neighbours to find the objects in the image. Finally, three feature extraction results are combined for efficient feature, which gives to the CNN for tumour classification. Fig. 5 shows the feature extraction of different brain tumour image.

\subsection{Classification using convolution neural network}

In traditionally, automatic classification of issue type of Region of Interest (ROI) plays significant role 
in CAD. Therefore SVM and CNN classifier is widely used to abnormal tumor classification [21], [22]. In this research, the input image and three set of feature values are given to CNN classifier for classifying abnormal bairn tumor such as meningioma, Glioma, pituitary tumor. The convolutional layer is a primary layer in the $\mathrm{CNN}$ classifier, it extracts the local information of an image such as texture, shape and some other features. Furthermore, conventional operation improves the input features and minimize the noise interference. In Eq. (11) expressed mapping operation in convolutional process.

$$
x_{j}^{l}=f_{c}\left(\sum_{i \in N j} x_{j}^{l-1} \times k_{i, j}^{l}+\theta_{j}^{l}\right)
$$

Here, $x_{j}^{l}$ is specified as the $j^{\text {th }}$ mapping set of convolutional layer $l, x_{j}^{l-1}$ is $i^{\text {th }}$ feature set denoting ( $l-1$ ) convolutional layer, $k_{i, j}^{l}$ is convolutional kernel between $i^{\text {th }}$ feature set and $j^{\text {th }}$ mapping set in $l$. Variable $\theta_{j}^{l}$ denoted as bias and $f_{c}$ is activation function. Next, the pooling process is used to reduce the possibility of over fitting during training process, which is expressed in Eq. (12).

$$
x_{j}^{l}=f_{P}\left(\beta_{j}^{l} \operatorname{down}\left(x_{j}^{l-1}\right)+\theta_{j}^{l}\right)
$$

Here, down(.) is down sampling approach from layer $(l-1)$ to $j^{\text {th }}, \theta_{j}^{l}$ and $\beta_{j}^{l}$ are denoted as additive bias and multiplicative bias. The final pooling layer (matrix features) are arranged to form a rasterization layer, which is further connected with fully connected layer. The output of node $j$ is expressed in Eq. (13).

$$
h_{j}=f_{h}\left(\sum_{i=0}^{n-1} w_{i, j} x_{j}-\theta_{j}\right)
$$

Here, $w_{i, j}$ is connection weight of input vector $x_{i}, \theta_{j}$ is denoted as node threshold. If the input layer deals with the multiclass issue, the softmax classifier is used in fully connected layer. Loss function of softmax classifier, which is expressed in Eq. (14).

$$
J(\theta)=-\frac{1}{n}\left[\sum_{i=1}^{n} \sum_{j=1}^{k} l\left\{y^{i}=j\right\} \log \frac{e^{\theta_{j}^{l}}}{\sum_{k} e^{\theta_{j}^{l}}}\right]
$$

Here, $e^{\theta_{j}^{l}}$ is input of $j^{\text {th }}$ in $l, \sum_{k} e^{\theta_{j}^{l}}$ is input of neurons, $\frac{e^{\theta_{j}^{l}}}{\sum_{k} e^{\theta_{j}^{l}}}$ is output of $j^{\text {th }}$ neuron. $e$ denoted as constant, $l($.$) Is indicator function. If the value in$ brace is true, output of indicator function is 1 . If the value in brace is false, the result of indicator is 0 . Next, add the rule item in $J(\theta)$ to prevent from falling into local optimum. Loss function of softmax classifier $J(\theta)$ after adding rule items, which is expressed in Eq. (15).

$$
\begin{gathered}
J(\theta)=-\frac{1}{n}\left[\sum_{i=1}^{n} \sum_{j=1}^{k} l\left\{y^{i}=j\right\} \log \frac{e^{\theta_{j}^{l}}}{\sum_{k} e^{\theta_{j}^{l}}}\right]+ \\
\frac{\rho}{2} \sum_{i=1}^{k} \sum_{j=0}^{m} \theta_{i j}^{2}
\end{gathered}
$$

Here, $\frac{\rho}{2} \sum_{i=1}^{k} \sum_{j=0}^{m} \theta_{i j}^{2}$ is weighted term that helps to stabilize the excessive parameters in training set.

\section{Result and discussion}

This section presents the experimental outputs of the tumour segmentation and classification methods and the comparative analysis to represent the effectiveness of the Hybrid KFCM-CNN method. The Hybrid KFCM-CNN method was implemented in MATLAB 2018b software tool with 4 GB Random Access Memory (RAM), Intel core processor, window 7 operating system. The proposed method was validated on T1-W CEMRI dataset. This dataset consists of 51 images with Glioma (25 images testing and 26 images training), 100 images with meningioma (25 images testing and 75 images training), and 100 images with pituitary tumours (25 images testing and 75 images training).

\subsection{Performance metrics}

The performance of the Hybrid KFCM-CNN method is compared in terms of accuracy, sensitivity, specificity and precision. The performance of segmentation is evaluated in terms of Dice and Jaccard. The performance metrics used for the experimentation are expressed in the following section.

To compute the dice coefficient is expressed in Eq. (16).

$$
\text { Dice Coefficient }=\frac{2 T P}{((2 T P+F P+F N))}
$$

Here, TP is True Positive, TN is True Negative, FN is False Negative, and FP is False Positive. Here, the dice coefficient value zero represents no similarity between the results and the value one denotes the similarity between the output and ground truth image. In Jaccard coefficient, the TP values are found by the overlaps between the segmented ground 
truth tumour labels and the machine generated tumour labels. The Jaccard coefficient is computed by using Eq. (17).

$$
\text { Jaccard coefficient }=\frac{T P}{F P+F N+T P}
$$

Accuracy is appropriate computation metrics for detecting the effectiveness of abnormality classification. The general formula of accuracy is given in Eq. (18).

$$
\text { Accuracy }=\frac{T P+T N}{T P+T N+F P+F N} \times 100
$$

The performance measurement is well defined as a relationship between input and output variable of proposed method by utilizing suitable performance parameters such as sensitivity and specificity. Common formula of sensitivity and specificity for abnormal tumor detection are expressed in Eq. (19) and Eq. (20).

$$
\begin{aligned}
& \text { Sensitivity or } T P R=\frac{T P}{T P+T N} \times 100 \\
& \text { Specificity or } T N R=\frac{T N}{T N+T P} \times 100
\end{aligned}
$$

Irrelevant images can be determined by utilizing Precision computation, which is expressed in Eq. (21).

$$
\text { Precision }=\frac{T P}{T P+F P} \times 100
$$

\subsection{Experimental result of hybrid KFCM - CNN method}

The T1 -W CEMRI dataset contains three classes of brain images, those are meningioma, Glioma and pituitary tumour. Here, the performance evaluation validated for one sample image in each class. The validation result showed that the proposed method outperformed compared to existing methodologies by means of dice, Jaccard accuracy, sensitivity and specificity and precision.

Table 1 shows the Dice and Jaccard coefficient comparison of Hybrid KFCM-CNN Method. The Dice similarity coefficient is known as similarity index, which calculates the overlapped region between the predicted area ground truth regions. For overall medical images, the average dice coefficient

\begin{tabular}{|c|c|c|c|c|c|}
\hline Input Image & TP & FP & $\mathbf{F N}$ & $\begin{array}{c}\text { Dice } \\
\text { Coefficient }\end{array}$ & $\begin{array}{c}\text { Jaccard } \\
\text { Coefficient }\end{array}$ \\
\hline $\begin{array}{c}\text { Class: } \\
\text { meningioma }\end{array}$ & 90 & 5 & 10 & 92.3077 & 85.7143 \\
\hline Class: Glioma & 95 & 15 & 8 & 89.2019 & 80.5085 \\
\hline Class: Pituitary & 85 & 12 & 18 & 85.000 & 79.9130 \\
\hline
\end{tabular}
of the proposed Hybrid KFCM-CNN method provides 88.8365 when compared with ground truth information.

Table 1. Performance of dice and Jaccard coefficient for Hybrid KFCM-CNN Method 
Table 2. Segmentation performance comparison of the existing and hybrid KFCM method

\begin{tabular}{|c|c|c|c|}
\hline Author & Segmentation methodology & Database & Accuracy (\%) \\
\hline $\begin{array}{c}\text { E. Abdel-Maksoud } \\
{[19]}\end{array}$ & $\begin{array}{c}\text { K-means Clustering integrated } \\
\text { with FCM system }\end{array}$ & Brain (DS1), (DS2), (DS3) & 96.83 \\
\hline Ali [20] & Morphological Pyramid and FCM & Brain Web (DS1), BRAST (DS2) & 97.05 and 95.85 \\
\hline Proposed & Hybrid KFCM & T1 -W CEMRI & 97.60 \\
\hline
\end{tabular}

Table 3. Performance of tumor classification by using Hybrid KFCM-CNN Method

\begin{tabular}{|c|c|c|c|c|}
\hline Tumour Class & Accuracy (\%) & Sensitivity (\%) & Specificity (\%) & Precision (\%) \\
\hline Meningioma & 97.33 & 88 & 100 & 100 \\
\hline Glioma & 100 & 100 & 100 & 100 \\
\hline Pituitary & 97.33 & 100 & 96 & 94 \\
\hline
\end{tabular}

Table 4. Classification performance comparison of the existing and hybrid KFCM-CNN method

\begin{tabular}{|c|c|c|c|}
\hline Author & $\begin{array}{c}\text { Classification } \\
\text { methodology }\end{array}$ & Database & Accuracy (\%) \\
\hline Cheng [21] & SVM & T1-W CEMRI & 91.14 \\
\hline Abiwinanda [22] & CNN & T1-W CEMRI & 84.16 \\
\hline Proposed & Hybrid KFCM-CNN & T1-W CEMRI & 97.33 \\
\hline
\end{tabular}

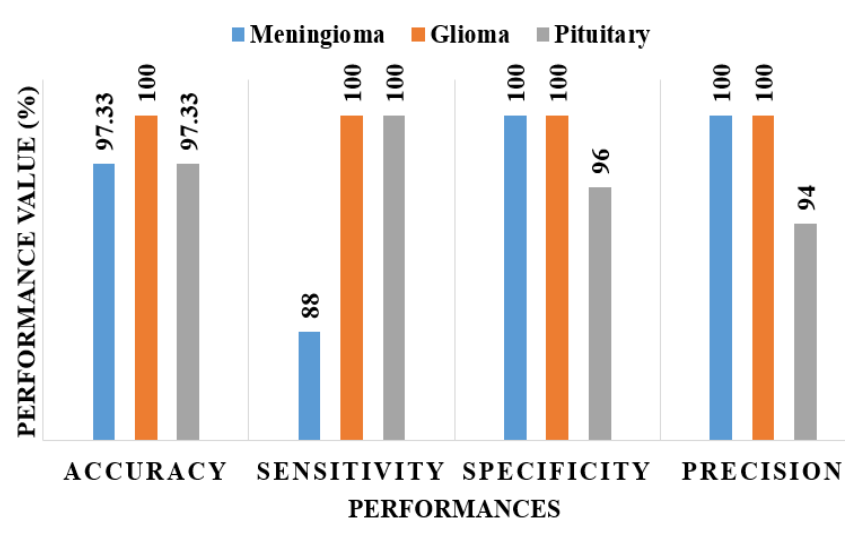

Figure. 6 Performance comparison for Meningioma, Glioma and Pituitary tumor classification

The average Jaccard coefficient of the Hybrid KFCM - CNN method delivers 82.045 when compared with the ground truth information. Table 2 shows the comparative analysis of proposed and existing tumour segmentation process.

The performance of segmentation process is compared to the two existing segmentation methods such as K-means clustering integrated with FCM system. The $\mathrm{K}$ - means clustering integrated with FCM system is obtained $96.83 \%$ of segmentation accuracy. The morphological pyramid and FCM achieved 97.05 and 95.85 of segmentation accuracy. The hybrid KFCM achieved $97.60 \%$ of segmentation accuracy compared to K-means clustering integrated with FCM system. The proposed Hybrid KFCM is obtained average $97.60 \%$ of segmentation accuracy for over all 3064 images. Table 1 confirmed that the proposed segmentation algorithm performs effectively than existing segmentation algorithms. Present a novel algorithm called Hybrid KFCM for fuzzy segmentation of MRI image data utilizing kernel included distance, image filtering and spatial membership. It is shown that the proposed algorithm has better segmentation outputs on simulated compared to existing FCM algorithms.

Performance evaluation of brain tumour classification by using Hybrid KFCM-CNN method is shown in Table 3. The CNN was trained on a brain tumour dataset consisting of 3064 T-1 weighted CE-MRI images. For Meningioma tumour class, the hybrid KFCM-CNN method achieved the $97.33 \%$ of accuracy, $88 \%$ of sensitivity, $100 \%$ of specificity, and $100 \%$ of precision.

For Glioma tumour class, Hybrid KFCM-CNN achieved, $100 \%$ of accuracy, $100 \%$ of sensitivity, $100 \%$ of specificity, and $100 \%$ of precision. The growth of pituitary tumour is abnormal in pituitary gland. For Pituitary tumour class, Hybrid KFCMCNN achieved $97.33 \%$ of accuracy, $100 \%$ of sensitivity, $96 \%$ of specificity, and $94 \%$ of precision. Fig. 6 shows the Comparison performance for Meningioma, Glioma and Pituitary classification.

Table 4 represents the comparative analysis of the tumour classification performance. In this research, the primary brain tumor is classified into three stages like Meningioma, Glioma and Pituitary by CNN method. Here, the tumor area is segmented using Hybrid KFCM. So, finally the percentage of accuracy of tumor detection shows for both segmentation and classification techniques. Extracted features are given to the CNN classifier for abnormal tumor classification, it can be efficiently improved the classification accuracy in this research. The gradient based CNN classifier extracts the both linear and nonlinear properties of tumor image and preserves 
quantitative relation between extracted features form segmented images. Here, the performance analysis confirms that the Hybrid KFCM-CNN method performs effectively in classification by means accuracy, sensitivity, specificity and precision. As shown in the Table 4, the proposed hybrid KFCMCNN represents the best classification performance compared to existing classifiers such as SVM and CNN.

It can be concluded that the Hybrid KFCM-CNN method possesses greater accuracy values compared to the existing methods for tumour segmentation and classification. Finally, the evaluation metrics confirmed that the proposed Hybrid KFCM-CNN method is more suitable for brain tumour detection and classification compared to existing methods in terms of accuracy, sensitivity, specificity, and precision.

\section{Conclusion}

Brain tumour detection is challenging task in Computer Aided Health Monitoring (CAHM) system. Image segmentation and classification plays an important role in medical image. In this research, the abnormal tumors were detected using MRI images. Because the MRI is more comfortable compared to CT images. Here, Hybrid KFCM was used for tumor segmentation. CNN was used for classification technique. By utilizing these techniques an efficient brain classification was constructed with maximum detection accuracy of $97.33 \%$. Simulation outputs using brain tumor database demonstrated the ability of proposed method for efficient segmentation and brain tumor classification. Ability of proposed Hybrid KFCM-CNN method was established on basis of obtained experimental results on brain tumor image database. The Hybrid KFCM-CNN method of tumour level classification is found to be efficient compared to existing methods through the analysis performed in terms of accuracy. Therefore, the proposed automatic segmentation and classification method is much suitable for brain tumour detection in the medical diagnosis field. In future work, an efficient bioinspired approach can further improve the performance and more datasets can be used for analysing the performance of the proposed scheme.

\section{References}

[1] D. Haritha, "Comparative study on brain tumour detection techniques", In: Proc. of International Conference on Signal Processing, Communication, Power and Embedded System, pp. 1387-1392, 2016.
[2] V. Viswa Priya and Shobarani, "An Efficient Segmentation Approach for Brain Tumor Detection in MRI", Indian Journal of Science and Technology, Vol.9, No.19, 2016.

[3] P.B. Kanade1 and P.P. Gumast, "Brain Tumor Detection Using MRI Images", International Journal of Innovative Research In Electrical, Electronics, Instrumentation and Control Engineering, Vol.3, No.2, 2015.

[4] A. Ari and D. Hanbay, "Deep learning based brain tumour classification and detection system", Turkish Journal of Electrical Engineering \& Computer Sciences, Vol.26, No.5, pp.2275-2286, 2018.

[5] P. Shanthakumar and P. Ganeshkumar, "Performance analysis of classifier for brain tumour detection and diagnosis", Computers \& Electrical Engineering, Vol.45, pp. 02-311, 2015.

[6] J. Naik and S. Patel, "Tumor detection and classification using decision tree in brain MRI", International Journal of Computer Science and Network Security, Vol.14, No. 6, pp.87, 2014.

[7] B. Devkota, A. Alsadoon, P.W.C. Prasad, A. K. Singh, and A. Elchouemi, "Image segmentation for early stage brain tumour detection using mathematical morphological reconstruction", Procedia Computer Science, Vol.125, pp.115123, 2018.

[8] B. Wilson and J.P. Malar Dhas, “An experimental analysis of Fuzzy C-means and K-means segmentation algorithm for iron detection in brain SWI using Matlab", International Journal of Computer Applications, Vol. 104, No. 15, 2014.

[9] V. Jumb, M. Sohani, and A. Shrivas, "Color image segmentation using K-means clustering and Otsu's adaptive thresholding", International Journal of Innovative Technology and Exploring Engineering, Vol.3, No.9, pp.72-76, 2014.

[10] V.K. Dehariya, S.K. Shrivastava, and R.C. Jain, "Clustering of Image Data Set Using K-Means and Fuzzy K-Means Algorithms", International conference on CICN, pp. 386- 391, 2010.

[11] A. Selvapandian and K. Manivannan, "Fusion based Glioma brain tumour detection and segmentation using ANFIS classification", Computer Methods and Programs in Biomedicine, Vol.166, pp.33-38, 2018.

[12] K.L. Hemalatha, H.N. Suresh, and S.K. Manvi, "Medical Image Segmentation Based on Extreme Learning Machine Algorithm in Kernel Fuzzy C-Means Using Artificial Bee Colony Method", International Journal of Intelligent Engineering and Systems, Vol.11, No. 6, pp.128136, 2018. 
[13] K.G. Satheesh and A.N. Joseph Raj, "Medical Image Segmentation and Classification Using MKFCM and Hybrid Classifiers", International Journal of Intelligent Engineering and Systems, Vol.10, No.6, pp. 9-19, 2017.

[14] Md. Sujan, N. Alam, S.A. Noman, and M. Jahirul Islam, "A Segmentation based Automated System for Brain Tumor Detection", International Journal of Computer Applications, Vol.153, No.10, pp. 41-49, 2016.

[15] D. Ravi, H. Fabelo, G. M. Callico, and G. Yang, "Manifold Embedding and Semantic Segmentation for Intraoperative Guidance with Hyperspectral Brain Imaging", IEEE Transactions on Medical Imaging, Vol.36, No.9, pp.1845-1857, 2017.

[16] A. Raju, P. Ratna, Suresh, and R. Rajeswara Rao, "Bayesian HCS-based multi-SVNN: A classification approach for brain tumour segmentation and classification using Bayesian fuzzy clustering", Biocybernetics and Biomedical Engineering, Vol.38, No.3, pp.646660, 2018.

[17] K. Usman and K. Rajpoot, "Brain tumour classification from multi-modality MRI using wavelets and machine learning", Pattern Analysis and Applications, Vol.20, No.3, pp.871-881, 2017.

[18] M. Soltaninejad, G. Yang, T. Lambrou, N. Allinson, T. L. Jones, T. R. Barrick, F. A. Howe, and $\mathrm{X}$. Ye, "Automated brain tumour detection and segmentation using superpixel-based extremely randomized trees in FLAIR MRI", International Journal of Computer Assisted Radiology and Surgery, Vol. 12, No.2, pp.183203, 2017.

[19] E. Abdel-Maksoud, M. Elmogy, and R. AlAwadi, "Brain tumour segmentation based on a hybrid clustering technique", Egyptian Informatics Journal, Vol.16, No.1, pp.71-81, 2015.

[20] H. Ali, M. Elmogy, E. El-Daydamony, and A. Atwan, "Multi-resolution mri brain image segmentation based on morphological pyramid and fuzzy c-mean clustering", Arabian Journal for Science and Engineering, Vol.40, No.11, pp.3173-3185, 2015.

[21] J. Cheng, W. Huang, C. Shuangliang, R. Yang, W. Yang, Z. Yun, Z. Wang, and Q. Feng, "Enhanced performance of brain tumour classification via tumour region augmentation and partition", PloS One, Vol.10, No.10, pp.e0140381, 2015.

[22] N. Abiwinanda, M. Hanif, S.T. Hesaputra, A. Handayani, and T. R. Mengko, "Brain Tumor
Classification Using Convolutional Neural Network", In: Proc. of World Congress on Medical Physics and Biomedical Engineering, pp. 183-189, 2019. 